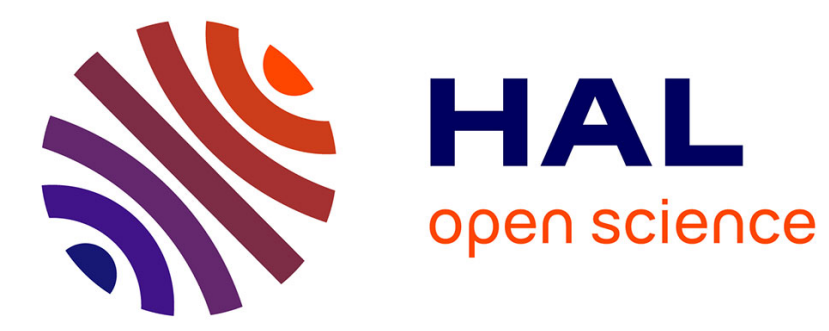

\title{
SUPERSPACE EMBEDDING OF 1-DIMENSIONAL QUASICRYSTALS
}

\author{
A. Janner
}

\section{To cite this version:}

A. Janner. SUPERSPACE EMBEDDING OF 1-DIMENSIONAL QUASICRYSTALS. Journal de Physique Colloques, 1986, 47 (C3), pp.C3-95-C3-102. 10.1051/jphyscol:1986309 . jpa-00225719

\section{HAL Id: jpa-00225719 https://hal.science/jpa-00225719}

Submitted on 1 Jan 1986

HAL is a multi-disciplinary open access archive for the deposit and dissemination of scientific research documents, whether they are published or not. The documents may come from teaching and research institutions in France or abroad, or from public or private research centers.
L'archive ouverte pluridisciplinaire HAL, est destinée au dépôt et à la diffusion de documents scientifiques de niveau recherche, publiés ou non, émanant des établissements d'enseignement et de recherche français ou étrangers, des laboratoires publics ou privés. 


\title{
SUPERSPACE EMBEDDING OF 1-DIMENSIONAL QUASICRYSTAIS
}

\author{
A. JANNER \\ Instituut voor Theoretische Fysica, Universiteit Nijmegen, \\ Toernooiveld, NL-6525 ED Nijmegen, The Netherlands
}

\begin{abstract}
Résumé - On considère le plongement dé quasicristaux à une dimension dans un espace: Euclidien bidimensionnel. Les conditions pour aboutir à des réseaux isométriques (carré et hexagonal) font apparatre oux paramètres qui caractérisent la classe d isomorphisme local, Ges mêmes paramètres dêterminent 1 a structure des modules libres engendrés par les composantes externes et celles jinternes des vecteurs réticulaires de l' espace direct et de celui réciproque. La Pyrrhotite $\mathrm{Fe}_{1-x} \mathrm{~S}$ est traitée comme exemple.
\end{abstract}

Abstract - The embedding of 1D quasicrystals in a $2 D$ Euclidean space is considered. The conditions for getting isometric lattices (square and hexagonaly involve two parameters characterizing the local isonorphism class. They also determine the structure of the free modules generated by the internal and the external components, respectively, of the lattice vectors in direct and in reciprocal space. Pyrrhotite $\mathrm{Fe} \mathrm{f}_{-\mathrm{x}} \mathrm{S}$ is treated as an example.

\section{I. - INTRODUCTION.}

The new class of crystal structures generaliy indicated by the name of quasicrystals shares the fundamental property of all other crystals known so far of having fourier wave vectors expressible as integral linear combination of a finite number of fundamental ones. When these basis wave vectors are linearly independent on the rationals but linearly dependent on the reals, the lattice periodicity is lost, the orystal is aperiodic (at least partially) and it is commonly called incommensurate. Quasicrystals belong to this class:

By bringing the fundamental wave vectors in 1-to-1 correspondence with the basis vectors of a lattice in a higher dimensional Euclidean space (called superspace), it is possible to embed the whole crystal structure and to recover space group symetry even in the incommensurate case. In present superspace approach the original structure is related to the embedded one by orthogonal projection in 
reciprocal space and intersection in direct space $/ 1 /, 12 \%$.

The crystallographic symmetry of quasicrystals has also been recovered by embedding in a higher dimensional Euclidean space following methods developed for the algebraic characterization of aperiodic tiling of the Penrose type $/ 3 /$. One of those methods, the so-called direct projection approach, is conceptually very similar to the superspace one, but involves projection and intersection in the reciprocal as well as in the direct space. It has been shown by Janssen that the two methods are actualiy equivalent 14/. In the superspace approach, quasicrystals appear as modulated crystals having discontinuous modulation lines and a basis structure not necessarily commensurate. Physically speaking, this can be expressed by saying that the Bragg reflection peaks of the diffraction pattern of a quasicrystal do not split (in general) into a subset of 'main reflections' on a reciprocal lattice and one of additional satellite reflections at incommensurate positions. Accordingly, quasicrystals represent a new class of incommensurate crystal structures. This opens the possibility of point group symmetries which are crystallographic in a higher dimensional space only, as it has been observed in icosahedral quasicrystals.

There is another new feature, not shared by all quasicrystals but typical for those of the Penrose type: that of being associated with an hypercubic lattice, despite the fact that the diffraction pattern in space never has the full hypercubic point group symmetry. The relevance of higher dimensional isometric lattices has always been a basic concern within the superspace approach because not occurring for incommensurate crystals with main reflections on lattice positions mapped onto themselves by symmetry transformations.

This paper is a preliminary investigation on the metrical relations one can associate to the embedding of an incommensurate crystal structure. The considerations are restricted to a 2D embedding of $1 D$ quasicrystals and the attention is focussed on lattice symmetry. The point group and the space group symmetry of the embedded structure, as well as the $2 \mathrm{D}$ and $3 \mathrm{D}$ quasiorystal case represent the next steps.

\section{II - SUPERSPACE EMBEDDING}

Considered is a $1 \mathrm{D}$ quasicrystal described by the density distribution:

$$
p(r)=\sum_{n_{1} n_{2}} \delta(1)\left(r-n_{1} a_{1}-n_{2} a_{2}\right) C\left(n_{1} n_{2}\right)
$$

where $n_{1}, n_{2}$ are integers, $a_{1}, a_{2}$ have an irrational ratio and $c\left(n_{1} n_{2}\right)$ is a characteristic function taking values 0 and 1 and ensuring a finite minimal distance between atomic positions as well as an infinite crystal extension. 
These conditions can be realized by the so-called direct projection method, where points of a $2 D$ lattice within a strip of width $D$ are projected perpendicularly to the 10 crystal line. A lattice basis $a_{15}, a_{2 s}$ then projects onto $a_{1}$ and $a_{2}$, respectively, and decomposes according to:

$$
a_{1 s}=\left(a_{1}, a_{1 I}\right), \quad a_{2 s}=\left(a_{2}, a_{2 I}\right)
$$

with $a_{i I}$ the so-called internal components, and $a_{i}$ the external ones. Choosing appropriate initial conditions one then has:

$$
C\left(n_{1} n_{2}\right)=\int d t \theta\left(D^{2} / 4-t\right) \delta^{(1)}\left(t-n_{1} a_{1 I}-n_{2} a_{2 I}\right)
$$

with $\theta$ a step function at $t=0$. In such a case the Fourier components of $\rho(r)$ are given by:

$$
\hat{\rho}_{\mathrm{FT}}(k)=\sum_{z_{1} z_{2} \delta}{ }^{(1)}\left(k-z_{1} a_{1}^{*}-z_{2} a_{2}^{*}\right) \hat{c}\left(z_{1} z_{2}\right)
$$

with $z_{1}, z_{2}$ integers and $a_{1}^{*}, a_{2}^{*}$ the (external) space components of the reciprocal basis vectors $a_{1 s}^{*}$ and $a_{2 s}^{*}$ :

$$
a_{\mu S}^{*}=\left(a_{\mu}^{*}, a_{\mu I}^{*}\right), \quad a_{\mu s}^{*} a_{\nu S}=\delta_{\mu \nu}, \quad \mu, \nu=1,2 .
$$

In eq. (4). $\hat{C}\left(z_{1} z_{2}\right)$ is the characteristic factor given by:

$$
\hat{C}\left(z_{1} z_{2}\right)=\frac{\sin \pi D\left(z_{1} a_{1 I}^{*}+z_{2} a_{2 I}^{*}\right)}{\pi D\left(z_{1} a_{1 I}^{*}+z_{2} a_{2 I}^{*}\right)} .
$$

From eq. (4) follows that such a quasicrystal satisfies the conditions for $z$-module crystallography $/ 5 /$.

Knowing the crystal structure and its diffraction pattern, the reciprocal free $Z$-module $M^{*}=\left\{a_{1}^{*}, a_{2}^{*}\right\}$ follows from the positions of the Bragg peaks; the direct one $M=\left\{a_{1}, a_{2}\right\}$ from the possible atomic positions. Furthermore, from the set of intensities described by $\hat{C}\left(z_{1} z_{2}\right)$ one gets $D_{1}^{*}$ and $D a_{2 I}^{*}$, and from the set of occupied atomic positions given by $c\left(n_{1} n_{2}\right)$ also $a_{1 I} / D$ and $a_{2 I} / D$. Accordingly, a quasicrystal structure determines in addition to $M$ and $M^{*}$ also the values of $a_{\mu I{ }^{*}{ }_{\nu I}}$ for $\mu, \nu=1,2, i . e$. the two real parameters $\sigma$ and $\rho$ defined by: 


$$
a_{1}^{*}=\sigma a_{2}^{*} \quad a_{2 I}^{*}=-\rho a_{1 I}^{*}
$$

From duality one gets the corresponding metrical relations in direct space:

$$
a_{1}=\rho a_{2} \quad \text { and } \quad a_{2 I}=-\sigma a_{1 I} \text {. }
$$

These parameters are the same as those considered by Socolar and Steinhardt $/ 6 /$. In their paper quasicrystals are considered having atomic positions at

$$
r_{n}=a\left(n+\alpha+\frac{1}{\rho}\left\lfloor\frac{n}{\sigma}+\beta\right\rfloor\right)
$$

with $\mathrm{n}$ integer, $\alpha, \beta$ and $\rho$ real, $\sigma$ irrational and $\lfloor\ldots\rfloor$ the greatest integer function. Denoting by $\theta$ the angle between $a_{1 s}$ and the external space direction, and by $\phi$ that between $a_{1 s}$ and $a_{2 s}$, the same positions can be expressed by:

$$
r_{n}=n a_{1 s} \cos \theta+\alpha+a_{2 s} \cos (\phi-\theta)\left\lfloor\frac{n a_{1 s} \sin \theta}{a_{2 s} \sin (\phi-\theta)}+\beta\right\rfloor,
$$

which implies eq.(8) for $a_{1}=a$. The additional parameters $\alpha$ and $\beta$ not considered above in the metrical relations for the lattice embedding do not modify the local isomorphism class of the quasicrystal, which is fixed by $\sigma$ and $\rho$. As explained in


between neighbouring atomic positions in the quasicrystal sequence. The only additional parameter (up to that of $a$ unit of length expressed by $a=1 / a^{*}$ ) appearing in the embedding can be indicated by $a_{1 I}=x a_{1}$. One thus arrives at the following canonical parametrization of the quasicrystal embedaing:

$$
\begin{aligned}
& a_{1 s}=a(1, \chi), \quad a_{2 s}=(1 / \rho,-\chi \sigma) \\
& a_{1 s}^{*}=\frac{a^{*}}{1+\sigma \rho}(\sigma \rho, 1 / \chi), \quad a_{2 s}^{*}=\frac{a^{*} \rho}{1+\sigma \rho}(1,-1 / \chi), \quad \text { with } a a^{*}=1 .
\end{aligned}
$$

\section{III - TWO DIMENSIONAL BRAVAIS LATTICES}

It is not self-evident that $2 \mathrm{D}$ Bravais lattices can be relevant for the embedding of 1D quasicrystals. Let us nevertheless consider the conditions imposed by the embedding into one of the five 2D Bravais lattices, disregarding in this exploratory paper the question of arithmetic equivalence (expressing the freedom in choosing the lattice basis).

No conditions are imposed by an Oblique lattice. Those for getting a Rectangular lattice can be expressed by $a_{1 \mathrm{~s}} \cdot a_{2 s}=0$, implying 


$$
x^{2}=\frac{1}{\sigma \rho}
$$

As one can see from eq.(9) one can choose $\sigma, p>0$ without loss of generality. Therefore any 1D quasicrystal as above admits a rectangular embedding. This is, however, not the end of the story, as in superspace embedding the description may influence the structural relations made explicit $/ 7 \%$.

The condition for getting a Diamond lattice can be cast into $a_{1 s}^{2}=a_{2 s}^{2}$ leading to the relation:

$$
\rho^{2} \chi^{2}=\frac{1-\rho^{2}}{1-\sigma^{2}} \text {, }
$$

implying that $\sigma^{2}$ and $\rho^{2}$ both have to be either larger or smaller than one. It appears that, if not already true, that condition can be managed by changing the sign of $\rho$, i.e. interchanging the role of short and long intervals. Therefore a di amond embedding is also always possibie.

One gets structural conditions in terms of $\sigma$ and $p$ only, for the embedding into the two isometric Bravais lattices, the square and the hexagonal one.

A Square lattice satisfies both eqs.(12) and (13) leading to the condition:

$$
\frac{\rho^{2}-1}{\rho}=\frac{\sigma^{2}-1}{\sigma}=\lambda
$$

implying

$$
\sigma=\rho .
$$

The parameter $\lambda$ in eq. (14) has been introduced because it appears in the corresponding characteristic equation determining the inflation rule of the quasicrystal (for $\lambda$ rational) $/ 8 /$. In the case of $\sigma=\rho$ the characteristic equation is given by:

$$
\sigma^{2}-\lambda \sigma-1=0 \text {, }
$$

which leads to the parametrization:

$$
\sigma=p=1 / 2\left(\lambda+\sqrt{\lambda^{2}+4}\right) \text {. }
$$

The corresponding inflation rule is $c_{n+1}=C_{n}^{\lambda} C_{n-1}$. For $\lambda=1$ one obtains the well-known Fibonacci tiling.

In the case of an Hexagonal lattice, one can consider it as rhombic with an angle $\phi=\pi / 3$, i.e. $a_{1 s} \cdot a_{2 s}=1 / 2 a_{1 s}^{2}=1 / 2 a_{2 s}^{2}$. This implies: 


$$
p=(\lambda+1) 0,
$$

with the parameter $\lambda$ appearing in the corresponding characteristic equation

$$
\rho^{2}+2 \lambda \rho-\lambda-1=0
$$

One then gets the structural condition for hexagonal lattice embedding:

$$
p=\frac{2 \sigma+1}{\sigma+2}
$$

The simplest solution is obtained for $\lambda=1$ giving the values $\sigma=1 / 2(-1+\sqrt{3})$ and $\rho$ $=2 \sigma$. Another similar solution follows for $\lambda=-1 / 2$ yielding $\sigma=(1+\sqrt{3})$ and $\rho=$ $1 / 2 \sigma$.

The discussion of the case $\phi=2 \pi / 3$ is quite analogous and results in a simple replacement of $\lambda$ by $-\lambda$, and a change of the sign of $\sigma$ and of $\rho$ in eq.(20).

\section{IV - MODULATED STRUCTURE}

Quasicrystals with atomic positions as in eq.(9) can also be expressed in terms of a basic structure and a modulation:

$$
r_{n}=n a\left(1+\frac{1}{\sigma \rho}\right)+\alpha-\frac{a}{\rho} \operatorname{frac}\left(\frac{n}{\sigma}+\beta\right)
$$

with $f r a c(x)$ the fractional part of $x$. Comparing eqs.(11) and (21) one sees that the reciprocal wave vectors of the basic structure are generated by $a_{1}^{*}=a^{*} \sigma \rho /(1+\sigma \rho)$, whereas the modulation wave vectors are generated by $q=\frac{1}{\sigma} a_{1}^{*}=a_{2}^{*}$. The $Z$-module $M^{*}$ is therefore expressible in terms of the same fundamental periodicities as in the previous description, as it should be.

From eq.(21) it follows furthermore that the lattice distance in the average structure (which is the basic structure also) is given by:

$$
A=\left(1+\frac{1}{\sigma \rho}\right) a=\frac{1}{A^{*}} .
$$

Consider now a rational approximation for $\sigma$, and denote, as conventional, the two spacings between neighbouring positions of the quasicrystal by:

$$
a=S \text { and } a(1+1 / \rho)=L \text {. }
$$

Then for the unit cell in the superstructure approximation one has: 


$$
n_{S} S+n_{L} L=\left(n_{S}+n_{L}\right) A, \quad n_{S} \text { and } n_{L} \text { integers. }
$$

One finds indeed that $\sigma$ expresses the relative frequency of $S$ and $L$ intervals

$$
\sigma=1+n_{S} / n_{L}
$$

\section{$V$ - AN EXAMPLE: PYRRHOTITE}

The crystal structure of Pyrrhotite, $\mathrm{Fe}_{1-\mathrm{x}} \mathrm{S}$ is that of a modulated crystal involving displacive as well as occupational modulation. The modulation wave vector $q$ is oriented along the hexagonal axis of the average structure and related to the compositional parameter $x$ in a simple way:

$$
q=2 x c^{*}=\frac{1}{N} c^{*}
$$

Such a compound is denoted as NC-Pyrrhotite. In general, it is thus an incommensurate crystal. Its $(3,1)$-dimensional superspace group symmetry has been determined, as well as the characteristics of the modulation waves $19 \%$. Here the displacive part only wiIl be considered. It appears that, within a good approximation, neighbouring $F e$ atoms along the c-direction involve two intervals only. Disregarding the other two crystallographic axes (lying in the hexagonal plane), Pyrrhotite can therefore be described as a 1D quasicrystal. In ref. 19/ the $\mathbf{N}=5.5$ case is considered in more detail; within the unit cell there are 9 long and 2 short intervals having ratio approximatively 1.0357. Therefore, for ensuring the conventions adopted above ( $a$ and $\rho$ positive) one has to choose $q=9 c^{*} / 11$ instead of $2 c^{*} / 11$ as in eq. $(26)$. One then gets the following set of parameters for the $5.5 \mathrm{C}$ Pyrrhotite:

$$
n_{S}=2, \quad n_{L}=9, \quad \sigma=11 / 9 \text { and } \rho-28 \text {. }
$$

Embedding into both the rectangular or the rhombic lattice is possible, but embedding into the isometric ones, square or hexagonal, is excluded. Indeed both the eqs.(16) and (20) are not satisfied. The same conclusion follows from the data one gets by choosing the $q=2 c^{*} / 11$ description.

\section{VI - FINAL REMARKS}

The standard embedding of a modulated structure as in eq.(21) is given by:

$$
a_{2}^{*}=\left(\sigma a_{2}^{*}, 0\right), \quad b_{s}^{*}=\left(a_{2}^{*}, b^{*}\right)
$$




$$
a_{s}=\left(1 / \sigma a_{2}^{*},-b / a\right), \quad b_{s}=(0, b), \quad \text { with } b^{*} b=1,
$$

so that only the reciprocal $Z$-module $M^{*}=a_{2}^{*}\{1, \sigma\}$ appears. On the contrary, the canonical quasicrystal embedding of eq.(11), takes the equivalent form:

$$
\begin{array}{ll}
a_{1 s}^{*}=\left(\sigma a_{2}^{*}, a_{1 I}^{*}\right), & a_{2 s}^{*}=\left(a_{2}^{*},-\rho a_{1 I}^{*}\right) \\
a_{1 s}=\left(\rho a_{2}, a_{1 I}\right), & a_{2 s}=\left(a_{2},-\sigma a_{1 I}\right)
\end{array}
$$

which reveals an intertwinning of direct and reciprocal space with respect to the internal and external components. These generate the following free modules:

$$
\begin{aligned}
& M^{*}=a_{2}^{*}\{1, \sigma\}, \quad M=a_{2}\{1, \rho\} \\
& M_{I}^{*}=a_{1 I}^{*}\{1, \rho\}, \quad M_{I}=a_{1 I}\{1, \sigma\} .
\end{aligned}
$$

showing the intertwinning.

ACKNOWLEDGEMENTS The very stimulating discussions with E. Springelkamp helped to clarify the role of diffraction intensities in the metrical embedding's conditions and are gratefully acknowledged.

\section{REFERENCES}

11/ De Wolff, P.M., Acta Cryst. A33 (1977) 609.

/2/ Janner, A. and Janssen, T., Phys. Rev. B15 (1977) 649.

/3/ De Bruijn, N.G., Proc. Kon. Ned. Ac. Wet. A84 (1981) 39.

14/ Janssen, T., "Crystallography of quasi-crystals", Acta Cryst. A (to appear).

15/ Janner, A., Ann. Israel Phys. Soc. $\underline{3}$ (1980) 118.

16/ Socolar, J.E.S. and Steinhardt, P.J., "Quasicrystals II: Unit Cell Configurations", (to appear).

/7/ Hogervorst, A.C.R., "Comparative Study of the Modulated Structures in $\mathrm{Rb}_{2} \mathrm{ZnBr}_{4}$ and in Related Compounds", Ph.D. Thesis, T.H. Delft, The Netherlands (1986).

18/. Bombieri, E. and Taylor Jean E., "Quasicrystal Tilings and Algebraic Number Theory", (to appear).

19/ Yamamoto, A. and Nakazawa, H., Acta Cryst. A38 (1982) 79. 